\title{
Dying tumor cell-derived exosomal miR- 194-5p potentiates survival and repopulation of tumor repopulating cells upon radiotherapy in pancreatic cancer
}

Ming-jie Jiang ${ }^{1,2+}$, Yi-yun Chen ${ }^{1 \dagger}$, Juan-juan Dai ${ }^{2 \dagger}$, Dian-na Gu${ }^{3}$, Zhu Mei ${ }^{2}$, Fu-rao Liu ${ }^{1,2}$, Qian Huang ${ }^{2,4}$ and Ling $\operatorname{Tian}^{1,2,5^{*}}$

\begin{abstract}
Background: Tumor repopulation is a major cause of radiotherapy failure. Previous investigations highlighted that dying tumor cells played vital roles in tumor repopulation through promoting proliferation of the residual tumor repopulating cells (TRCs). However, TRCs also suffer DNA damage after radiotherapy, and might undergo mitotic catastrophe under the stimulation of proliferative factors released by dying cells. Hence, we intend to find out how these paradoxical biological processes coordinated to potentiate tumor repopulation after radiotherapy.

Methods: Tumor repopulation models in vitro and in vivo were used for evaluating the therapy response and dissecting underlying mechanisms. RNA-seq was performed to find out the signaling changes and identify the significantly changed miRNAs. QPCR, western blot, IHC, FACS, colony formation assay, etc. were carried out to analyze the molecules and cells.
\end{abstract}

Results: Exosomes derived from dying tumor cells induced G1/S arrest and promoted DNA damage response to potentiate survival of TRCs through delivering miR-194-5p, which further modulated E2F3 expression. Moreover, exosomal miR-194-5p alleviated the harmful effects of oncogenic HMGA2 under radiotherapy. After a latent time, dying tumor cells further released a large amount of PGE2 to boost proliferation of the recovered TRCs, and orchestrated the repopulation cascades. Of note, low-dose aspirin was found to suppress pancreatic cancer repopulation upon radiation via inhibiting secretion of exosomes and PGE2.

Conclusion: Exosomal miR-194-5p enhanced DNA damage response in TRCs to potentiate tumor repopulation. Combined use of aspirin and radiotherapy might benefit pancreatic cancer patients.

Keywords: Tumor repopulation, Tumor repopulating cell, Exosome, microRNA, DNA damage response, Aspirin, Radiotherapy, Pancreatic cancer

\footnotetext{
* Correspondence: TL09168@hotmail.com

${ }^{+}$Ming-jie Jiang, Yi-yun Chen and Juan-juan Dai contributed equally to this work.

'Institute of Translational Medicine, Shanghai General Hospital, Shanghai Jiao Tong University School of Medicine, Shanghai 201620, China

${ }^{2}$ Shanghai Key Laboratory of Pancreatic Diseases, Shanghai General Hospital, Shanghai Jiao Tong University School of Medicine, Shanghai 201620, China Full list of author information is available at the end of the article
}

\section{Introduction}

Cancer radiotherapy is one of the main strategies of cancer treatment, and more than half of cancer patients receive radiotherapy to cure localized disease, palliate symptoms, or control disease in incurable cancers [1]. However, in a small set of cancers such as pancreatic cancer, the effects of radiotherapy remain unsatisfactory

(c) The Author(s). 2020 Open Access This article is licensed under a Creative Commons Attribution 4.0 International License, which permits use, sharing, adaptation, distribution and reproduction in any medium or format, as long as you give appropriate credit to the original author(s) and the source, provide a link to the Creative Commons licence, and indicate if changes were made. The images or other third party material in this article are included in the article's Creative Commons licence, unless indicated otherwise in a credit line to the material. If material is not included in the article's Creative Commons licence and your intended use is not permitted by statutory regulation or exceeds the permitted use, you will need to obtain permission directly from the copyright holder. To view a copy of this licence, visit http://creativecommons.org/licenses/by/4.0/. The Creative Commons Public Domain Dedication waiver (http://creativecommons.org/publicdomain/zero/1.0/) applies to the data made available in this article, unless otherwise stated in a credit line to the data. 
[2], which contributes to the fact that pancreatic cancer ranks one of the most malignant cancers with the lowest 5 -year survival rate among all kinds of cancers [3]. As one of the "5Rs" of radiobiology, tumor repopulation is taken for a major cause of treatment failure [4]. Our previous works revealed that tumor repopulation significantly occurred in pancreatic cancer after radiation [57]. Nevertheless, its underlying mechanisms largely remain unclear.

Recent researches showed that chemoradiation-caused dying tumor cells promoted tumor repopulation $[5,8]$. The irradiated dying tumor cells underwent significant intracellular signaling changes and secreted large amounts of molecules. It was reported that the lipid metabolite PGE2 secreted by dying tumor cells promoted tumor repopulation [8]. Moreover, dying tumor cells also secreted HMGB1 [9], VEGF [10], etc. to accelerate proliferation of the admixed surviving tumor cells, promote angiogenesis, and facilitate cancer cell repopulation and metastasis [5].

As known, the secretions of dying tumor cells are complex, and usually comprise cytoplasmic soluble factors, organelles and their contents, and extracellular vesicles (EVs) $[9,11,12]$. Notably, EVs, especially exosomes, are important mediators of cell-cell communications and play vital roles in promoting cancer progression [13]. Moreover, microRNAs (miRNAs), a type of small non-coding RNA, are vital contents of EVs. Loading of miRNAs into exosomes is not a random process, and the selective enrichment of miRNAs are tightly regulated such as by KRAS mutation [14]. Cancer cell-secreted exosomal miRNAs were found to involve in stroma cell reprogramming [15] and pre-metastatic niche formation [16]. And exosomal miRNAs from cancer stroma cells were reported to confer chemoresistance [17]. However, little is known about whether exosomes are involved in tumor repopulation.

Besides, tumor repopulating cells (TRCs) are supposed to be the major cells for tumor repopulation, and exert some cancer stem-like cell (CSC) properties [18, 19]. Yet, TRCs just represent a functional distinction. It is still unclear whether there're any relative markers to define TRCs. Some markers, such as c-MET, CD44, CD133, LGR5, ALDH1, etc. are used to identify pancreatic CSCs [20]. Do these markers also apply to identifying TRCs in pancreatic cancer? Further, TRCs also suffer from radiation as dying cells do and sustain DNA damages in radiotherapy. Cell cycle progression with DNA damage could induce mitotic catastrophe, which is the main form of cell death induced by ionizing radiation (IR) [21]. Considering that large amounts of proliferation stimuli are released by irradiated dying tumor cells, it is important to figure out how TRCs survive and are stimulated to fast proliferation under IR-induced damages. This study aims to delineate how TRCs survive and repopulate after radiotherapy, and seeks appropriate agents to intervene pancreatic cancer repopulation.

Herein, we first reported that exosomal miR-194-5p derived from radiation-caused dying tumor cells potentiated tumor repopulation. We found that irradiated dying tumor cells released a large amount of exosomes in the early phase after radiation. These exosomes further enhanced DNA damage responses to promote survival of TRCs that were characterized as $\mathrm{ALDH}^{+}$cells. Next generation sequencing of exosomal miRNAs identified miR-194-5p as the significantly elevated miRNA. We further found that miR-194-5p could downregulate the transcription factor E2F3 to induce cell cycle arrest and contribute to repairing the damaged TRCs. Moreover, the transfer of miR-194-5p to TRCs might alleviate the harmful effects of HMGA2 under radiotherapy. Subsequently, dying tumor cells released PGE2 to accelerate proliferation of the repaired TRCs. More importantly, low-dose aspirin was found to suppress tumor repopulation via inhibiting the secretion of exosomes and PGE2. Our data provides new insights into tumor repopulation and suggests a simple and feasible intervention method for pancreatic cancer.

\section{Results \\ Exosomes released from irradiated dying tumor cells dynamically potentiate tumor repopulation}

We firstly examined the biological processes of tumor repopulation after radiotherapy. In tumor repopulation model in vitro, where irradiated dying tumor cells (feeder cells) were co-cultured with the admixed living tumor cells (reporter cells) as described before [8], we found that dying tumor cells accelerated proliferation of reporter cells after 3 days (Additional file 1: Fig. S1a). However, the proliferation of reporter cells was slightly inhibited when cocultured for 1 day (Additional file 1: Fig. S1b). To further clarify the timepoint of cell cycle changes, we applied a transwell co-culture system, where the upper layer was plated with irradiated or unirradiated feeder cells and the lower layer was viable reporter cells. It was showed that irradiated dying tumor cells induced G1/S cell-cycle arrest in reporter cells $12 \mathrm{~h}$ after radiation, but the effect was reversed $36 \mathrm{~h}$ after radiation (Additional file 1: Fig. S1c). Further, when reporter cells were cultured with conditioned media $(\mathrm{CM})$ from irradiated dying tumor cells, similar proliferation-inhibitory effects were shown (Additional file 1: Fig. S1d). These data indicate that secretions from irradiated dying tumor cells inhibit cell proliferation at the immediate early phase after radiation but promote proliferation in the later phase.

To figure out why dying tumor cells inhibited proliferation of reporter cells transiently after radiation, transcriptome sequencing of irradiated dying tumor cells was performed. Gene Ontology analysis revealed that 
signaling concerning EVs and exosomes regulation were enriched in irradiated cells (Fig. 1a; Additional file 2: Fig. S2a). We further confirmed the increased release of exosomes in CM of irradiated pancreatic cancer cells compared with unirradiated ones (Fig. 1b). These exosomes were well characterized by reference methods (Additional file 2: Fig. S2b-d). Moreover, proteins involving in exosome processing were also upregulated in irradiated cells as well as in patient-derived xenograft (PDX) mouse model of pancreatic cancer (Additional file 2: Fig. S2d-g). Further, dying tumor cell-derived exosomes were used to treat reporter cells. Exosomes from irradiated dying tumor cells induced G1/S cell cycle arrest, inhibited EdU incorporation and proliferation of the treated cells (Fig. 1c-e; Additional file 2: Fig. S2h-i). Moreover, exosomes-depleted CM of irradiated cells lost the inhibitory effects (Fig. 1e). In vivo administration of irradiated dying tumor cells-derived exosomes also suppressed proliferation of the subcutaneous tumors as indicated by BrdU incorporation assay (Fig. 1f). These data indicate that the proliferation inhibitory effect is largely due to dying cell-derived exosomes.

To verify the direct role of exosomes in surviving tumor cells, we performed colony formation assay in vitro. Colony formation ability of cancer cells treated with dying tumor cell-derived exosomes was significantly enhanced after radiation (Fig. 1g). Moreover, GW4869, an SMase inhibitor that was widely used for reducing exosome secretion [22], dramatically inhibited colony formation of pancreatic cancer cells after irradiation (Additional file 2: Fig. S2j). Meanwhile, GW4869 showed no effects on colony formation of the unirradiated cells (Additional file 2: Fig. S2k). These results indicate that exosomes derived from dying tumor cells can promote cell survival after radiation.

We further explored the role of exosomes in tumor repopulation in PDX mouse models. Irradiated PDX tumors showed accelerated repopulation after a lag phase, even though a little variance existed among tumors derived from different patients (Fig. 1h; Additional file 2: Fig. S2l-m). Surprisingly, when PDX mice were simultaneously treated with GW4869, tumor repopulation was significantly slowed down (Fig. $1 \mathrm{~h}$; Additional file 2 : Fig. S2l-m), and the survival time of tumor-bearing mice was prolonged (Fig. 1i). Moreover, much more Ki-67 ${ }^{+}$ cells resided in irradiated tumors, which was significant reduced by simultaneous GW4869 treatment (Fig. 1j). These results indicate that dying tumor cells-derived exosomes promote tumor repopulation.

\section{Dying tumor cells-released exosomes potentiate survival} of the ALDH1A $1^{+}$TRCs

We supposed that TRCs might act as the primary recipients of exosomes. As currently no marker was applied to identify TRCs, we thus collected surviving pancreatic cancer cells after radiation and analyzed the expression profiles of cancer stem cell-related genes by qPCR array. It was identified that 62 of the 84 target genes were upregulated compared with the unirradiated controls (Additional file 3: Fig. S3a). Especially, 10 of the 11 statistically significantly changed genes were upregulated (Fig. 2a; Additional file 3: Fig. S3a). Moreover, the ratio of CD24 $4^{+}$cells and CD326 (ESA) ${ }^{+}$cells were significantly upregulated in irradiated PANC-1 cells (Additional file 3: Fig. S3b). These data indicate that surviving cells exhibit higher CSC properties.

Remarkably, the expression of ALDH1A1 was significantly upregulated after radiation (Fig. 2a; Additional file 3: Fig. S3a). As known, ALDH1A1 was one of the most important markers of pancreatic cancer stem-like cells [23]. The ALDEFLOUR ${ }^{\mathrm{Tx}}$ experiment revealed that the ratio of $\mathrm{ALDH}^{+}$cells was significantly elevated in surviving cancer cells and primary pancreatic tumor cells after radiation (Fig. 2b-c; Additional file 3: Fig. S3c). qPCR assay and western blot demonstrated that the expression of ALDH1A1 was upregulated in irradiated pancreatic cancer cells and PDX tumor tissues (Fig. 2d; Additional file 3: Fig. S3d-e). Immunohistochemistry (IHC) staining further showed that ALDH1A1 ${ }^{+}$cells were highly enriched after irradiation in PDX tumor tissues (Fig. 2e). However, the accumulation of ALDH1 $\mathrm{A1}^{+}$cells was significantly inhibited by GW4869 (Fig. 2e), which indicated that exosomes might promote survival of the ALDH1 $11^{+}$cells.

To investigate how the ratio of $\mathrm{ALDH} 1 \mathrm{Al}^{+}$cells increased after irradiation, we constructed a lineage tracing system, in which GFP expression indicated ALDH1A1 expression after being induced by 4-hydroxytamoxifen (4OHT) (Additional file 3: Fig. S3f). The ALDH1A1 expression was elevated in $\mathrm{GFP}^{+}$cells (ALDH1A1 ${ }^{+}$cells) than in DsRed $^{+}$cells (ALDH1A1 ${ }^{-}$cells) (Additional file 3: Fig. S3g). The colony-forming capacity of $\mathrm{GFP}^{+}$cells also improved in soft agar and in plate after irradiation compared with DsRed $^{+}$cells (Additional file 3: Fig. S3h-i). Notably, GFP ${ }^{+}$ cells were significantly enriched after irradiation, while additional treatment with GW4869 significantly suppressed the enrichment of $\mathrm{GFP}^{+}$cells (Fig. 2f). Meanwhile, a higher proportion of $\mathrm{GFP}^{+}$cells were also raised in subcutaneous tumors after radiation (Additional file 3: Fig. S3j).

Furthermore, we examined the direct protective role of dying tumor cells on TRCs. Irradiated $\mathrm{GFP}^{+}$cells exhibited significantly higher survival rate when they were cocultured with irradiated pancreatic cancer cells or treated with CM thereof (Fig. 2g-h). As shown, exosome-depleted $\mathrm{CM}$ of irradiated cells lost the protective effect, while the isolated exosomes significantly promoted survival of irradiated $\mathrm{GFP}^{+}$cells (Fig. 2h). These results indicate that the ALDH1A1 ${ }^{+}$cells represent a subpopulation of TRCs, whose survival is 


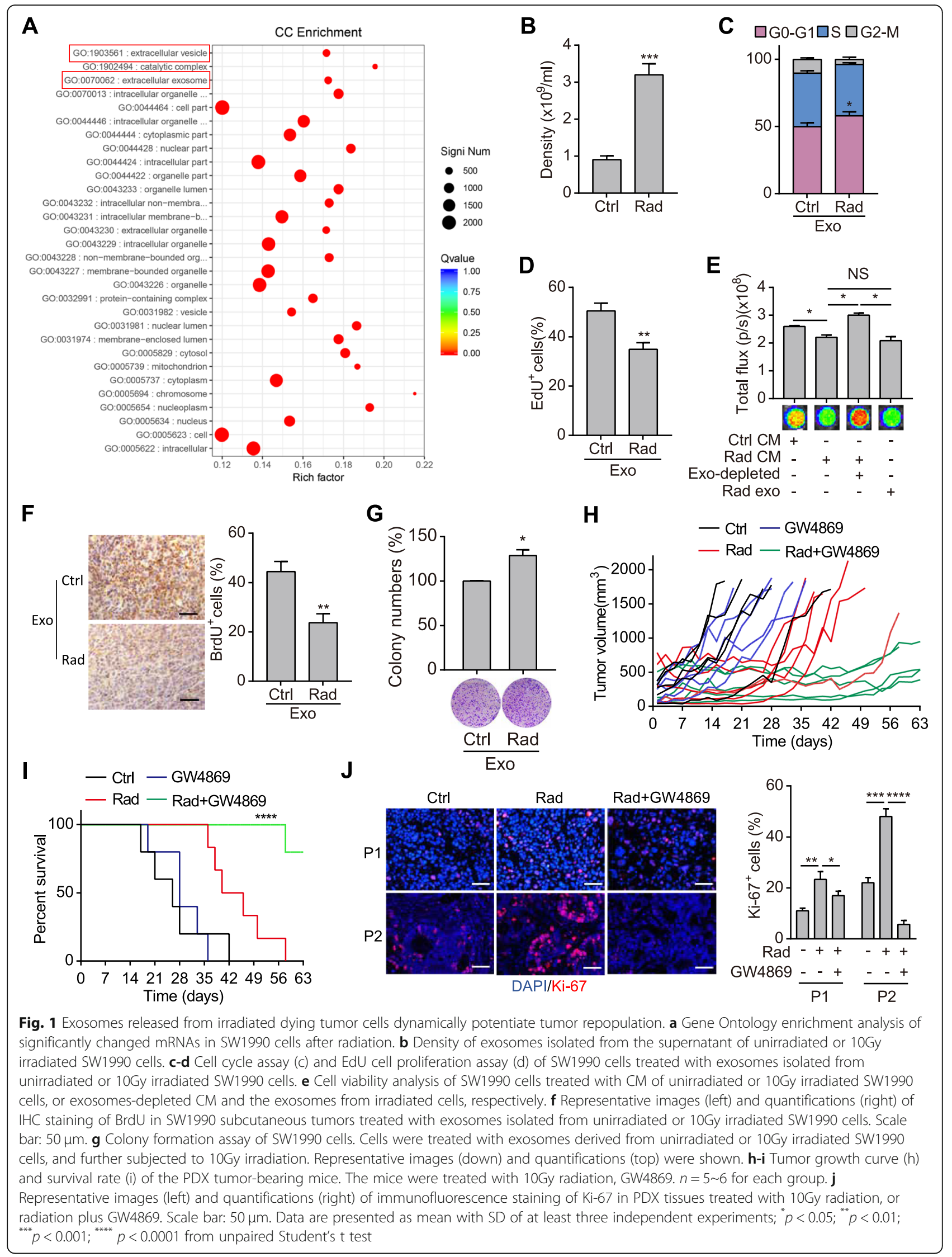




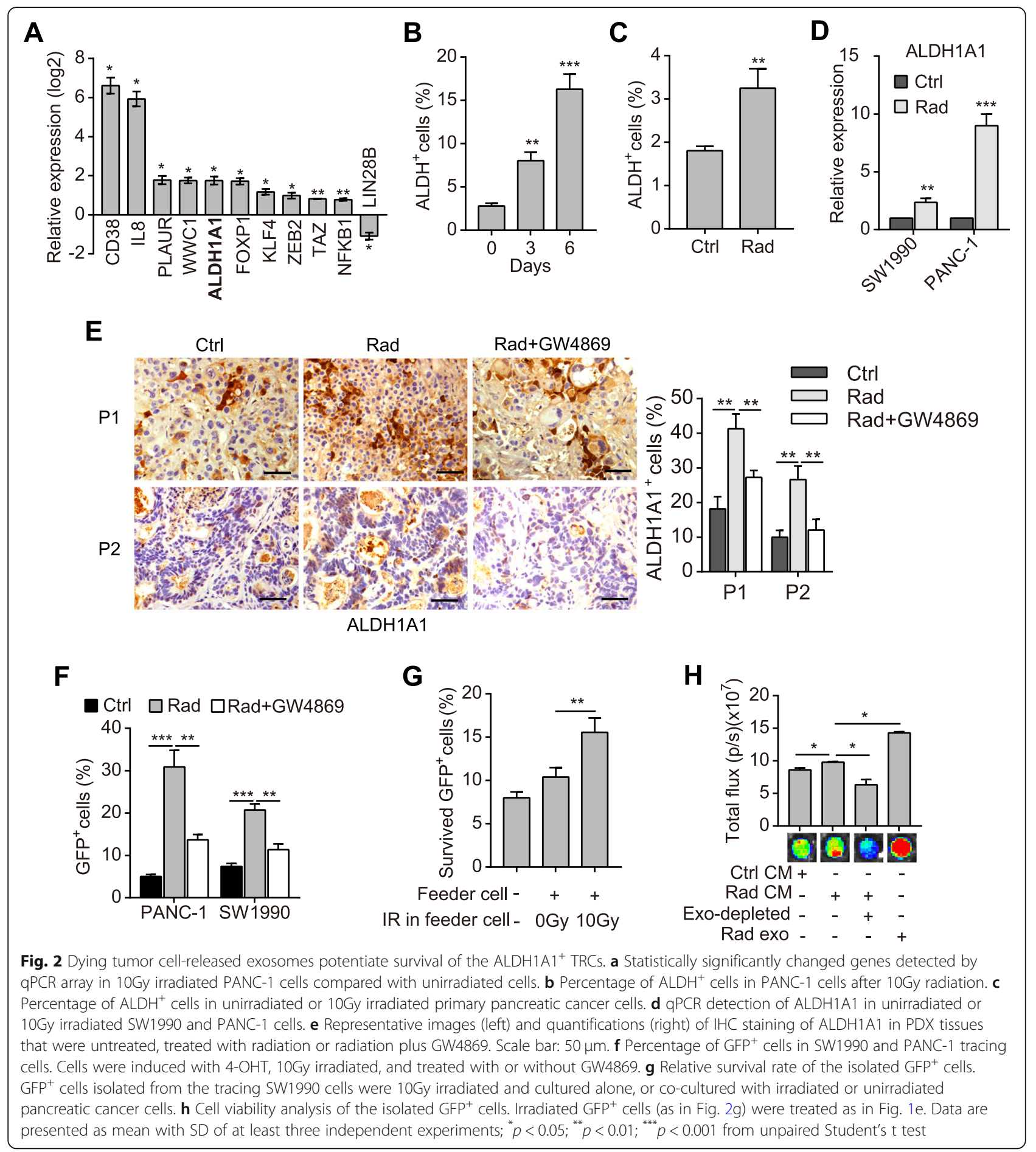

enhanced by dying tumor cell-derived exosomes under radiotherapy.

\section{Exosomes potentiate survival of TRCs via promoting DNA damage response}

We then explored how dying tumor cell-derived exosomes promoted survival of the ALDH1A $1^{+}$cells. H\&E staining showed that cells in the PDX tumors presented enlarged nuclei with significant heteromorphosis 14 days after radiation (Fig. 3a), which indicated the accumulation of radiation damage. 50 days after radiation, cells recovered, and "normal" cell nuclei morphology was returned. However, the recovery of tumor cells was hindered by GW4869 (Fig. 3a). Notably, ALDH1A1 ${ }^{+}$cells 


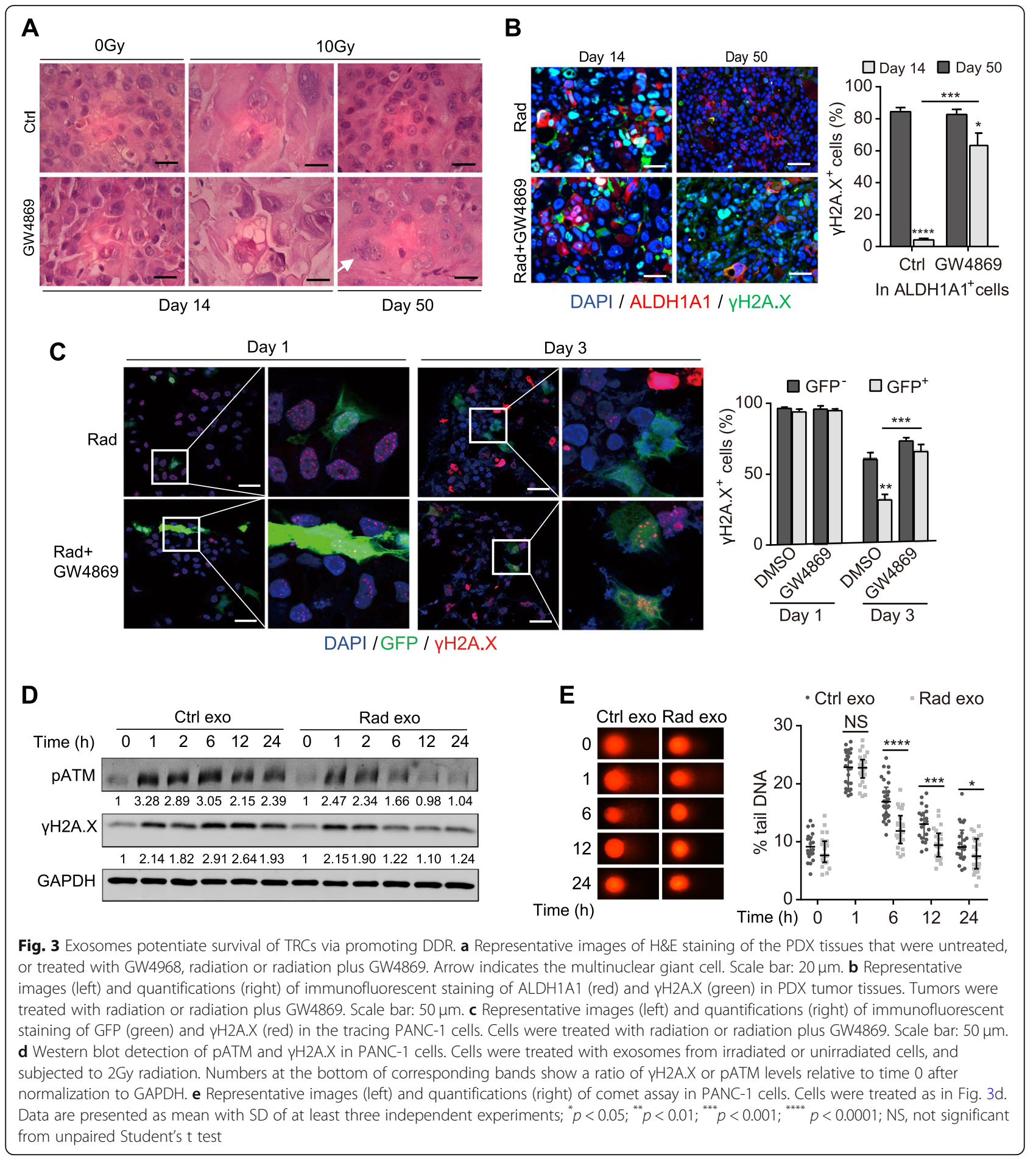

also suffered from DNA damage after radiation as indicated by the double staining of ALDH1A1 and $\gamma \mathrm{H} 2 \mathrm{~A} . \mathrm{X}$ (Fig. 3b). And in the GW4869 treatment group, majority of ALDH1A1 ${ }^{+}$cells still manifested significant $\gamma \mathrm{H} 2 \mathrm{~A} . \mathrm{X}$ positive foci 50 days after radiation (Fig. 3b). These data indicate that the survival of TRCs after radiation involves DNA damage response (DDR), which is modulated by exosomes from irradiated dying tumor cells.

Further, we testified whether exosomes directly promote DDR for survival of irradiated TRCs in vitro. As showed in the tracing cells, irradiation caused DNA damage in both $\mathrm{GFP}^{+}$and $\mathrm{GFP}^{-}$cells (Fig. 3c). The 
DNA damages in $\mathrm{GFP}^{+}$cells were rapidly repaired after radiation as evidenced by the faster elimination of $\gamma$ H2A.X positive foci. GW4869 significantly impaired the repair of $\mathrm{GFP}^{+}$cells, for most of them still carried $\gamma \mathrm{H} 2 \mathrm{~A}$.X positive foci 3 days after radiation (Fig. 3c). Furthermore, exosomes derived from irradiated dying tumor cells significantly accelerated attenuation of $\gamma$-H2A.X and pATM (Fig. 3d) and reduction of DNA damage (Fig. 3e) in irradiated cells. Collectively, these results demonstrate that irradiated dying tumor cells-derived exosomes potentiate the survival of TRCs via promoting DDR.

\section{Exosomal miR-194-5p promotes repair of the damaged TRCs}

As important cargos in exosomes, we inferred that miRNAs might play important roles in survival of the TRCs. So, we performed miRNA-seq and found that two validated miRNAs, miR-196b-5p and miR-194-5p, were significantly upregulated in dying tumor cells-derived exosomes (Fig. 4a). Interestingly, as a P53-induced miRNA, miR-194 might involve in genome stability [24]. miR-194-5p upregulation in exosomes derived from irradiated dying tumor cells was validated by qPCR assay (Fig. 4b). miR-194-5p expression was also upregulated in exosomes derived from the plasma of irradiated PDX mice (Fig. 4c), as well as in several pancreatic cancer cell lines after irradiation (Additional file 4: Fig. S4a).

Transfection of miR-194-5p mimics induced G1/S cell cycle arrest and suppressed EdU incorporation (Additional file 4: Fig. S4b-c), similar to the effect of exosomes from dying tumor cells. Moreover, stable overexpression of miR-194-5p significantly suppressed colony formation ability (Additional file 4: Fig. S4d). These results indicate that miR-194-5p suppresses pancreatic cancer cell proliferation. In addition, miR-194-5p overexpression also inhibited pancreatic cancer cell migration and invasion (Additional file 4: Fig. S4e-f).

However, miR-194-5p mimics-transfected cells exerted more powerful capabilities of colony formation after radiation (Fig. 4d). Further, a tet-on system was constructed to overexpress miR-194-5p under the control of doxycycline (Additional file 4: Fig. S4g). The colony formation of pancreatic cancer cells was dramatically inhibited when doxycycline was used (Additional file 4: Fig. S4h). However, if pancreatic cancer cells were exposed to radiation after doxycycline induction, colony formation of these cells would be significantly enhanced (Fig. 4e). Besides, miR-194-5p mimics significantly promoted attenuation of $\gamma \mathrm{H} 2 \mathrm{~A} . \mathrm{X}$ and pATM (Fig. 4f) and reduction of DNA damage (Additional file 4: Fig. S4i) in irradiated pancreatic cancer cells. These results indicate that exosomal miR-194-5p facilitates the survival of TRCs via promoting DDR.
We further identified the main effective targets of miR-194-5p. With the miRNA target prediction algorithms, 474 potential targets of miR-194-5p were predicted by Targetscan, 289 targets by Pictar, and amongst 87 targets were identical (Additional file 5: Fig. S5a). KEGG annotation revealed that only the transcription factor E2F3 in the predicted targets was related to cell cycle regulation (Additional file 5: Fig. S5a). E2F3, a member of E2F family, was widely revealed to promote cancer initiation and progression [25, 26]. The dualluciferase reporter assay proved E2F3 as the direct target of miR-194-5p (Fig. 4g). E2F3 expression was inhibited by miR-194-5p mimics and miR-194-5p stably overexpression in pancreatic cancer cells, while slightly elevated by miR-194-5p inhibitor (Additional file 5: Fig. S5b-c). Contrary to miR-194-5p, E2F3 overexpression promoted cell cycle progression and EdU incorporation (Additional file 5: Fig. S5d-e), and hindered attenuation of pATM and $\gamma \mathrm{H} 2 \mathrm{~A} . \mathrm{X}$ (Fig. 4h) and reduction of DNA damage (Additional file 5: Fig. S5f) in irradiated pancreatic cancer cells. E2F3 knockout elevated the colony formation ability of pancreatic cancer cells after radiation, and transfection of miR-194-5p mimics didn't further enhance the ability (Fig. 4i). These data indicate that exosomal miR-194-5p inhibits E2F3 to induce G1/S arrest and promote DNA damage repair.

Previous investigations identified HMGA2 and E2F3 as two of the eight key regulator hubs of pancreatic cancer [27]. The expression of HMGA2 was positively correlated with E2F3 in pancreatic cancer (Additional file 6: Fig. S6a). Unexpectedly, it was reported that HMGA2, an oncogene with pro-cancer stemness ability, interacted with miR-194 [28]. We found that HMGA2 negatively regulated miR194-5p expression (Fig. 4j). HMGA2 and miR-194-5p were also revealed to be reversely correlated in pancreatic cancers by TCGA database analysis (Additional file 6: Fig. S6b).

HMGA2 expression was significantly upregulated in pancreatic cancer tissues compared with the normal pancreas tissues (Additional file 6: Fig. S6c). ALDH1A1 ${ }^{+}$ pancreatic cancer cells also showed enhanced HMGA2 expression (Additional file 6: Fig. S6d). Meanwhile, HMGA2-overexpressed cells exhibited more stem celllike features as shown by the higher proportion of $\mathrm{ALDH}^{+}$cells (Additional file 6: Fig. S6e) and the greater ability of soft-agar colony formation (Additional file 6: Fig. S6f). HMGA2 overexpression also promoted proliferation of pancreatic cancer cells (Additional file 6: Fig. S6g), while HMGA2 knockout significantly inhibited their colony formation ability (Additional file 6: Fig. S6h). Besides, HMGA2 knockout significantly inhibited the migration and invasion of pancreatic cancer cells (Additional file 6: Fig. S6i-j). These data demonstrate that HMGA2 is enriched in the stem-like ALDH1A1 ${ }^{+}$ cells and contributes to pancreatic cancer progression. 


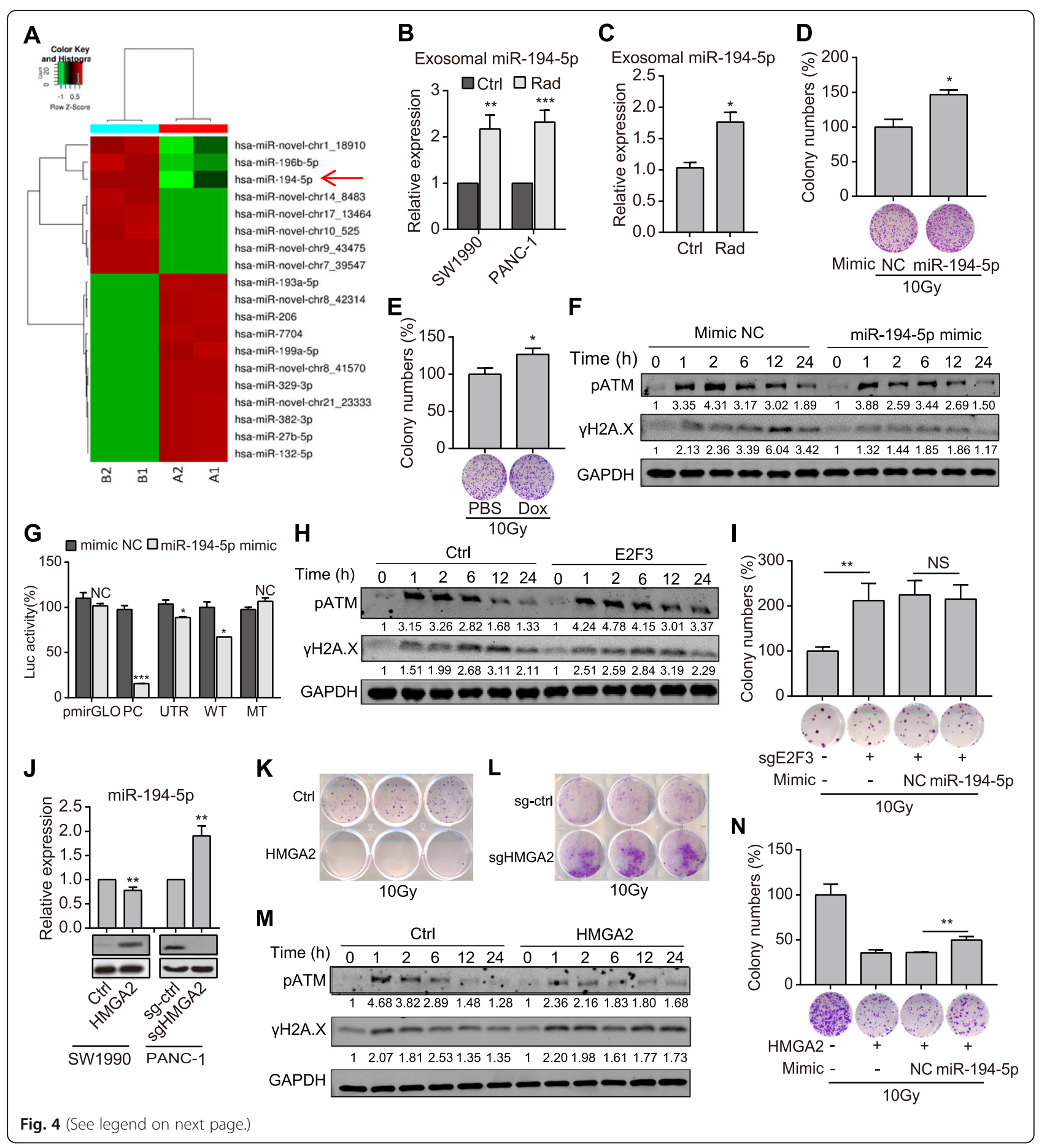


(See figure on previous page.)

Fig. 4 Exosomal miR-194-5p promotes repair of the damaged TRCs. a Heatmap of the significantly changed miRNAs in exosomes isolated from irradiated (group B) or unirradiated pancreatic cancer cells (group A). b-c qPCR detection of exosomal miR-194-5p. Exosomes were isolated from either irradiated/unirradiated cells (b) or plasma of PDX tumor-bearing mice (c). d-e Relative colony numbers (top) and representative images (down) of SW1990 cells (d) or SW1990 tet-on cells (e) that were treated as indicated. $\mathbf{f}$ Expression of pATM and YH2A.X in PANC-1 cells transfected with miR-194 mimics or mimic NC and subjected to 2Gy radiation. Numbers at the bottom of corresponding bands show a ratio of YH2A.X or pATM levels relative to time 0 after normalization to GAPDH. $\mathbf{g}$ Dual luciferase assay to identify E2F3 as the direct target of miR-194-5p in PANC-1 cells. The construction of reporter plasmids was shown in Fig. S9. $\mathbf{h}$ Expression of PATM and YH2A.X expression in the indicated PANC1 cells treated with 2Gy radiation. Numbers are as described in Fig. 4f. i Colony formation assay of SW1990 cells that were treated as indicated. $\mathbf{j}$ qPCR analysis of the expression of miR-194-5p (top) and western blot analysis of the expression of HMGA2 (down) in pancreatic cancer cells. $\mathbf{k}-\mathbf{I}$ Colony formation assay of the indicated SW1990 ( $k$ ) and PANC-1(I) cells after 10Gy radiation. $\mathbf{m}$ Expression of pATM and $\gamma$ H2A.X in the indicated SW1990 cells treated with 2Gy irradiation. Numbers are as described in Fig. 4f. $\mathbf{n}$ Colony formation assay of SW1990 cells treated as indicated. Data are presented as mean with SD of at least three independent experiments; ${ }^{*} p<0.05 ;{ }^{* *} p<0.01 ;{ }^{* * *} p<0.001$; NS, not significant from unpaired Student's t test

However, when pancreatic cancer cells were exposed to $10 \mathrm{~Gy}$ radiation, overexpression of HMGA2 dramatically inhibited the colony formation ability of these cells (Fig. 4k), while knock-out of HMGA2 enhanced cell survival and colony formation (Fig. 4l). HMGA2 also hindered attenuation of pATM and $\gamma \mathrm{H} 2 \mathrm{~A} . \mathrm{X}$ (Fig. 4m) and reduction of DNA damage (Additional file 6: Fig. S6k) in irradiated pancreatic cancer cells. Importantly, the elevated HMGA2 expression in $\mathrm{GFP}^{+}$cells was not significantly reduced after radiation (Additional file 6: Fig. S6l). Coincidentally, transfection of miR-194-5p in the HMGA2-overexpressed cancer cells partially enhanced the colony formation ability, and colonies of the miR194-5p transfected group were significantly larger than that of the control (Fig. $4 \mathrm{n}$ ). Together, these data indicate that the enriched HMGA2 in TRCs promotes their stemness and cancer progression but is potentially detrimental to the survival and recovery of irradiated TRCs, while exosomal miR-194-5p from dying tumor cells transiently reverses the effects of HMGA2 under radiotherapy to preserve the TRCs.

\section{Dying tumor cell-released PGE2 promotes proliferation of the recovered TRCs}

As described earlier, next to the proliferation inhibitory effects at the immediate early phase after radiation, dying tumor cells accelerated the proliferation of reporter cells (Additional file 1: Fig. S1), which implied that the recovered TRCs were driven to accelerate proliferation by some secretions other than aforementioned exosomal miR-194. As known, irradiated dying tumor cells also released PGE2, a lipid metabolite that was deemed to be a vital mediator of tumor repopulation after chemoradiation [8, 29]. Thus, we quantified PGE2 in the CM of irradiated dying pancreatic cancer cells by ELISA, and found that the contents of PGE2 remained unchanged in the CM at $24 \mathrm{~h}$ after radiation, yet slightly elevated at $48 \mathrm{~h}$ and significantly increased at $72 \mathrm{~h}$ (Fig. 5a). The change of PGE2 contents were in line with the proliferation dynamics of reporter cells cocultured with irradiated cells (Additional file 1: Fig. S1).

Corresponding to PGE2, the expression of PTGS2 (COX-2), a key enzyme for PGE2 biogenesis, also underwent a short lag phase before rapidly increased after radiation (Fig. 5b-c; Additional file 7: Fig. S7). In contrast, the expression of exosome-related proteins, such as RAB27A, TSG101 and CD9, increased immediately after radiation in pancreatic cancer cells (Fig. 5c; Additional file 7: Fig. S7b). Of note, when the expression of COX-2 gradually increased, the expression of exosome-related proteins decreased (Fig. 5c; Additional file 7: Fig. S7b). In the PDX mouse model, IHC staining also showed that the expression of exosome-related proteins was highly elevated soon after radiation till declined 12 days later, when the expression of COX-2 started to upregulate significantly (Fig. 5d).

Meanwhile, it was found that tumor cell repopulation was significantly restrained by celecoxib, a COX-2 inhibitor that inhibited PGE2 synthesis, in a dosedependent manner (Fig. 5e). Although PGE2 was found to accelerate the proliferation of tumor cells, addition of PGE2 to tumor cells immediately after radiation reversely inhibited cell viability (Fig. 5f). Of note, celecoxib treatment didn't influence the expression of proteins concerning exosomes processing (Fig. 5g). These results demonstrate that the secretions from irradiated dying tumor cells are released in a time-course manner, which are vital for tumor repopulation.

Together, these results indicate that dying tumor cellreleased exosomes and PGE2 orchestrate tumor repopulation cascades, of which exosomal miR-194-5p firstly inhibits cell proliferation to promote recovery of TRCs, and then PGE2 boosts accelerated proliferation of the recovered TRCs.

Aspirin suppresses tumor repopulation after radiotherapy via impairing the secretions from dying tumor cells Our previous discussion prompted that aspirin might have the potential to suppress tumor repopulation in 


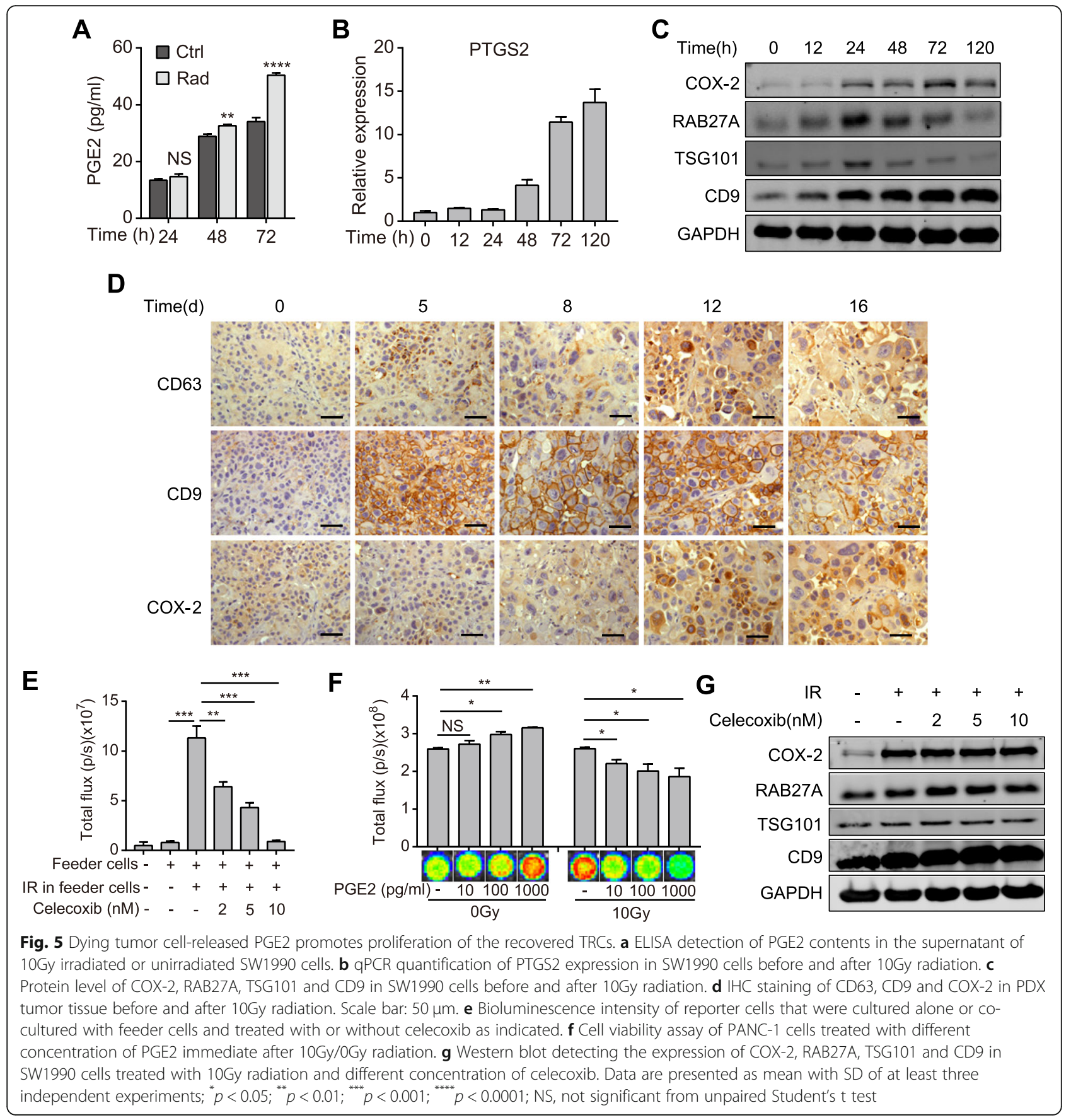

pancreatic cancer [30]. And aspirin was also found to influence exosomes and miRNAs [31]. So, we firstly tested the effect of aspirin treatment in tumor cell repopulation model in vitro. Aspirin dramatically restrained the accelerated proliferation of reporter cells cocultured with irradiated dying tumor cells (Fig. 6a), while had no effect on the proliferation of reporter cells when they were cultured alone (Additional file 8: Fig. S8a). Aspirin reduced PGE2 contents in the supernatant of irradiated pancreatic cancer cells (Fig. 6b), and decreased the quantity of exosomes released from these cells (Fig. 6c). Besides, miR-194-5p was downregulated in exosomes derived from aspirin-treated irradiated cells (Fig. 6d). Moreover, compared with exosomes derived from cells treated with radiation alone, exosomes derived from the aspirin-treated irradiated cells lost the ability to induce G1/S arrest and reduce EdU incorporation (Fig. 6e-f). Accordingly, exosomes derived from the aspirin-treated 


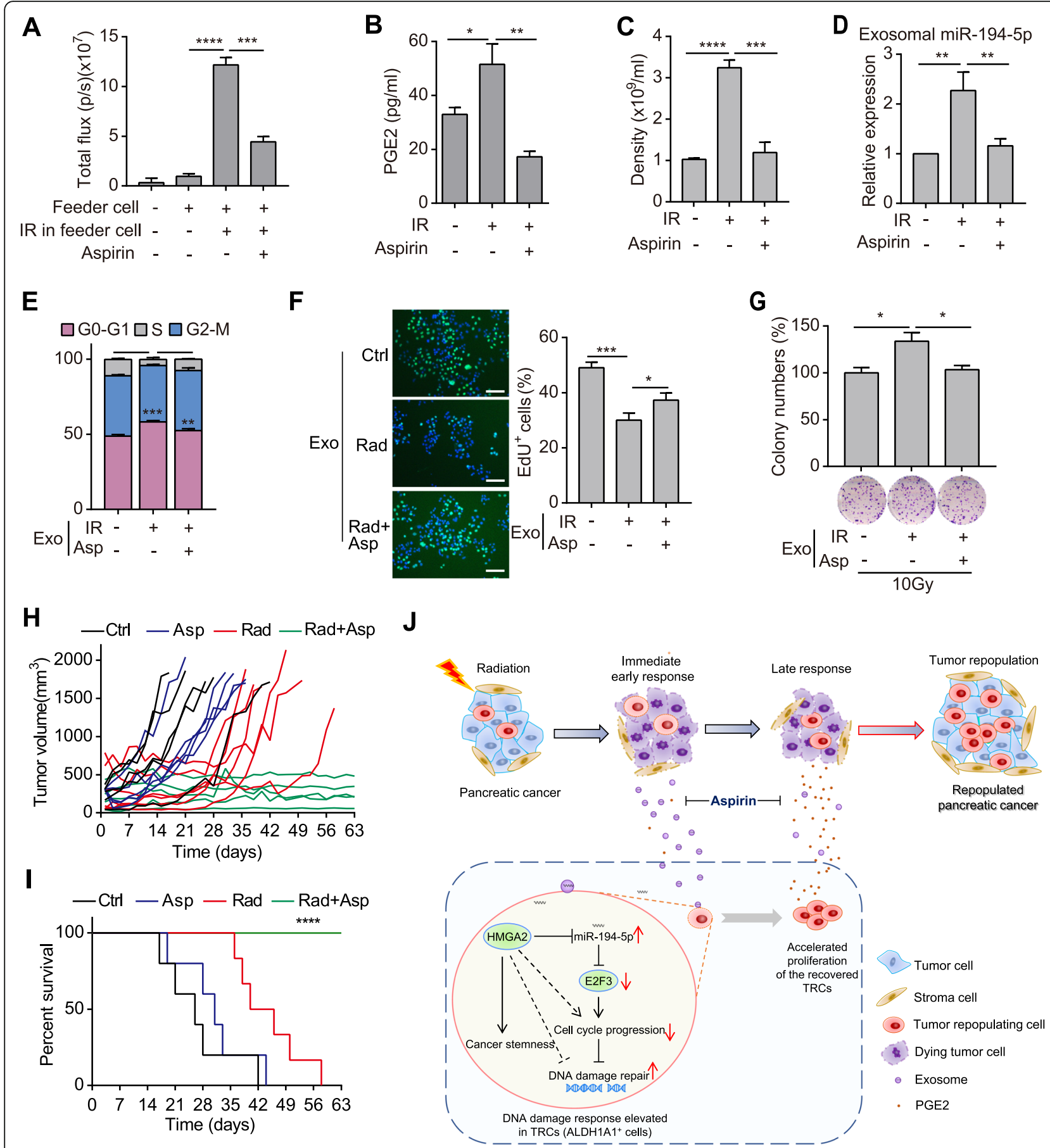

Fig. 6 Aspirin suppresses tumor repopulation after radiotherapy via impairing the secretions from dying tumor cells. a Bioluminescence intensity of reporter cells that were cultured alone or co-cultured with feeder cells and treated with or without aspirin as indicated. $\mathbf{b}$ ELISA detecting the concentration of PGE2 in the supernatant of SW1990 cells that were treated with 10Gy irradiation or irradiation plus aspirin. c Density of exosomes released from SW1990 cells that were treated as in Fig. 6b. d qPCR detection of miR-194-5p in exosomes isolated from SW1990 cells treated as in Fig. 6b. e-f Cell cycle assay (e), representative images (left) and quantifications (right) of EdU incorporation (f) in SW1990 cells treated with exosomes from the indicated cells. Scale bar: $100 \mu \mathrm{m}$. g Relative colony numbers (top) and representative images (down) of SW1990 cells treated with exosomes from the indicated cells and subjected to 10Gy radiation. $\mathbf{h}-\mathbf{i}$ Tumor growth curve (h) and survival rate (i) of PDX tumorbearing mice that were untreated, or treated with aspirin, 10Gy radiation or radiation plus aspirin. $n=5 \sim 6$ for each group. $\mathbf{j}$ Schematic diagram showing that aspirin disrupts dying tumor cell-orchestrated tumor repopulation cascades. Data are presented as mean with SD of at least three independent experiments; $p<0.05 ;{ }^{* * *} p<0.01 ;{ }^{* * *} p<0.001 ;{ }^{* * * *} p<0.0001$ from unpaired Student's $t$ test, expect in Fig. 6i, which was calculated by Log-rank (Mantel-Cox) test 
irradiated cells couldn't promote colony formation of pancreatic cancer cells after radiation (Fig. 6g).

We then tested the effects of aspirin treatment in PDX mouse models. Aspirin monotherapy had no therapeutic effects on PDX tumors, while radiotherapy alone showed marginally beneficial effects (Fig. 6h-i). However, combination of aspirin treatment and radiotherapy dramatically suppressed tumor repopulation and prolonged the survival time of tumor-bearing mice (Fig. 6h-i). Moreover, aspirin impaired the recovery of tumor tissues after radiotherapy (Additional file 8: Fig. S8b-c), which was similar to the effects of GW4869. Further, aspirin also suppressed the enrichment of ALDH1 $11^{+}$TRCs and reduced the proportion of $\mathrm{Ki}-$ $67^{+}$cells in tumor tissues after radiotherapy (Additional file 8: Fig. S8d-e). These data indicate that aspirin suppresses tumor repopulation after radiotherapy in pancreatic cancer.

\section{Discussions}

Tumor repopulation is one of the " $5 \mathrm{Rs"} \mathrm{of} \mathrm{radiobiology}$ and the main cause of treatment failure [4]. We herein propose that radiation-induced dying tumor cells orchestrate tumor repopulation. The irradiated dying cells firstly secret exosomes to induce cell cycle arrest and promote DNA damage repair to potentiate the survival of ALDH1 $\mathrm{A1}^{+}$TRCs, and then release large amounts of PGE2 to boost proliferation of the recovered TRCs. Lowdose aspirin could restrain secretion of exosomes and PGE2 from dying tumor cells, and suppress tumor repopulation after radiation in pancreatic cancer (Fig. 6j).

TRCs has been suggested to be the main cells responsible for tumor repopulation $[18,19]$. However, TRCs also suffer from radiation in radiotherapy. As known, the main mechanism of radiotherapy is to induce DNA damage and cause cell death. The induction of DNA damage is accompanied by DDRs characterized by cellcycle arrest and DNA damage repair. Moreover, the signaling of DDR such as P53 are mutated with high frequency in cancers, which has been exploited to enhance the efficacy of cytotoxic therapy or develop synthetic lethality strategies such as PARP inhibitors. Thus, to survive under radiotherapy, TRCs have to develop some additional mechanisms to compensate the impaired DDRs, such as getting help from dying cells.

Actually, it is a common phenomenon that dying cells promote survival of the tissue stem cells in different organisms. In Arabidopsis, chilling stress-induced programmed cell death in columella stem cell daughters protected stem cells through maintaining auxin maximum [32]. In Drosophila, irradiated dying cells were found to protect stem cells from death and keep them for regeneration, which was called the "Mahakali effect" [33]. In human, therapy-sensitive cells could promote survival and proliferation of the residue surviving tumor cells in targeted therapy [34]. We here have revealed that the "dying-for-surviving" phenomenon also exists in radiotherapy.

In addition to radiotherapy, other cytotoxic therapies such as chemotherapy or photodynamic therapy also enhanced the secretion of EVs from tumor cells [35]. EVs are also found to promote survival of stem cells by other studies. EV-packaged Wnt ligands derived from macrophage could rescue intestinal stem cells and enhance their survival after radiation [36]. Mesenchymal stromal cell-derived EVs also rescued radiation-damaged murine marrow hematopoietic cells via transferring miRNAs [37]. We here report that exosomes from irradiated dying tumor cells transfer miR-194-5p to the residual TRCs and facilitate their recovery. Nevertheless, it remains unclear why the secretion of exosomes elevates in irradiated dying tumor cells. Further investigations are needed.

Particularly, we here report that miR-194-5p is the mediator to enhance DDR and TRC survival in pancreatic cancer. Previous investigations also demonstrated that irradiated tumor cell-derived exosomes promoted radioresistance of the recipient cells via transferring miR-208a or miR-1246, which were found to promote cell proliferation in lung cancer $[38,39]$. As far as we known, we are the first one to report that an exosometransmitted proliferation inhibitory miRNA promotes radioresistance. As a P53-responsive miRNA, miR-194 was revealed to induce cell cycle arrest $[24,40]$. CRISPR-Cas9 library-based screening revealed that miR194-5p was a pro-fitness miRNA in Hela cells [41]. These data are in line with our findings. However, sustained cell cycle arrest may cause tumor suppression. Indeed, higher expression of miR-194-5p was associated with better prognosis in pancreatic cancer [42]. We also found that sustained overexpression of miR-194-5p inhibited cell proliferation, migration, invasion and colony formation. However, miR-194 was also reported to contribute to tumor growth and progression in pancreatic cancer [43]. Thus, the function of miR-194-5p might be highly context-dependent. Notably, exosomal miR194-5p might performed like a "firefighter" in a way under radiotherapy, which was transferred from dying tumor cells to rescue TRCs in a short time. Once DNA damage was repaired, exosomal miR-194-5p was withdrawn, leaving no time for miR-194-5p to suppress the proliferation of TRCs. Accordingly, the lipid metabolite PGE2 were released from dying tumor cells, and took over to boost repopulation of the repaired TRCs. Moreover, exosomal miR-194 was also revealed to activate PMVECs and promote angiogenesis [44], which could potentiate tumor repopulation. Additionally, it was also found that HMGA2 attenuated DNA damage repair [45, 
46], and E2F3 mediated DNA damage-induced apoptosis [47]. Our findings coincide with these data that both genes promote cancer progression but inhibit DNA damage repair.

Finally, low-dose aspirin was found to suppress tumor repopulation in pancreatic cancer after radiotherapy via inhibiting secretion of exosomes and PGE2 from dying tumor cells. These findings indicate that aspirin might have a good application prospect in radiotherapy. As an agent that has pleiotropic effects [30], aspirin might have some other mechanisms to repress tumor repopulation. Thus, more investigations should be carried out in the future to identify the specific approach of aspirin in repressing tumor repopulation. It was found that aspirin could reverse tumor-promoting inflammation and contribute to evoking tumor-inhibitory immunity [48], which indicated that aspirin might help to arouse the potential of immunogenic cell death, which could be induced by radiotherapy but often hindered $[49,50]$. As a safe and widely available prescribed medicine, it is convenient to test the efficacy of aspirin in the clinic under appropriately designed clinical study.

\section{Conclusion}

In summary, we found that dying tumor cells potentiated survival of the TRCs and promoted their repopulation in pancreatic cancer via sequentially secreting exosomes, which delivered miR-194-5p, and PGE2. Our data implied that combination treatment of radiotherapy and aspirin might greatly benefit pancreatic cancer patients.

\section{Supplementary information}

Supplementary information accompanies this paper at https://doi.org/10. 1186/s12943-020-01178-6.

Additional file 1: Figure S1. Irradiated dying tumor cells dynamically regulate proliferation of reporter cells.

Additional file 2: Figure S2. Increased secretion of exosomes from dying tumor cells inhibit cell proliferation but potentiate tumor repopulation.

Additional file 3: Figure S3. $\mathrm{ALDH}_{1 \mathrm{~A}} 1^{+}$cells are a subpopulation of TRCs with cancer stem cell-like properties.

Additional file 4: Figure S4. miR-194-5p suppresses pancreatic cancer cell proliferation, migration and invasion, but promotes DNA damage repair.

Additional file 5: Figure S5. E2F3 is a target of miR-194 and promotes pancreatic cell proliferation, inhibits DNA damage repair.

Additional file 6: Figure S6. HMGA2 promotes pancreatic cancer stemness and progression, but inhibits DNA damage repair.

Additional file 7: Figure S7. Expression of PTGS2 and exosome-related proteins in PANC-1 cells after radiation.

Additional file 8: Fig. S8. Effects and mechanisms of aspirin in suppressing pancreatic cancer repopulation.

Additional file 9: Fig. S9. Schematic diagram of the plasmid constructs. Additional file 10. Supplementary materials and methods.

Additional file 11: Table S1. Oligos and primers used in this study.

\section{Abbreviations}

4-OHT: 4-hydroxytamoxifen; CM: Conditioned media; CSC: Cancer stem-like cell; DDR: DNA damage response; EV: Extracellular vesicle;

IHC: Immunohistochemistry; IR: lonizing radiation; miRNA: microRNA;

PDX: Patient-derived xenograft; TRC: Tumor repopulating cell

\section{Acknowledgements}

We thank Dr. Yang-yang Hu, Dr. Jingbo Xiao, and Ms. Chen-yun Dai for technical supports, and Mr. Tao Lang for laboratory services.

\section{Authors' contributions}

MJ performed the experiments, analyzed the data and wrote the manuscript. YC, JD did the experiments and analyzed the data. DG, ZM, FL did some experiments and provided useful helps. QH gave valuable suggestions. LT designed and supervised the study, explained the data and wrote the manuscript. All authors read and approved the final manuscript.

\section{Funding}

This study was supported by the funding of the National Natural Science Foundation of China (No. 81773073 and No. 81802328), Young Talents Program of the First Affiliated Hospital of Wenzhou Medical University (No. qnyc094) and Wenzhou Science and Technology Plan Project (No. Y20180214).

\section{Availability of data and materials}

All data generated or analyzed during this study are included in this published article and its supplementary information files. The datasets generated during the current study are available in the GEO repository (GSE138983 and GSE138984).

\section{Ethics approval and consent to participate}

Human pancreatic cancer specimens were obtained according to a protocol approved by the institutional review board of the Shanghai General Hospital and written informed consent was obtained from all patients for the use of their samples. All animal experiments were conducted according to protocols approved by Shanghai General Hospital's Institutional Animal Care and Use Committee (IACUC).

\section{Consent for publication}

Not applicable.

\section{Competing interests}

The authors declare that they have no competing interests.

\section{Author details}

'Institute of Translational Medicine, Shanghai General Hospital, Shanghai Jiao Tong University School of Medicine, Shanghai 201620, China. ${ }^{2}$ Shanghai Key Laboratory of Pancreatic Diseases, Shanghai General Hospital, Shanghai Jiao Tong University School of Medicine, Shanghai 201620, China. ${ }^{3}$ Department of Chemoradiotherapy, The First Affiliated Hospital of Wenzhou Medical University, Zhejiang 325000, China. ${ }^{4}$ Cancer Center, Shanghai General Hospital, Shanghai Jiao Tong University School of Medicine, Shanghai 201620, China. ${ }^{5}$ Department of Central Laboratory, Shanghai Chest Hospital, Shanghai Jiao Tong University School of Medicine, Shanghai 200030, China.

Received: 12 November 2019 Accepted: 5 March 2020

Published online: 30 March 2020

\section{References}

1. Thariat J, Hannoun-Levi JM, Sun Myint A, Vuong T, Gerard JP. Past, present, and future of radiotherapy for the benefit of patients. Nat Rev Clin Oncol. 2013;10:52-60.

2. Heestand GM, Murphy JD, Lowy AM. Approach to patients with pancreatic cancer without detectable metastases. J Clin Oncol. 2015;33:1770-8.

3. Siegel RL, Miller KD, Jemal A. Cancer statistics, 2019. CA Cancer J Clin. 2019; 69:7-34.

4. Kim JJ, Tannock IF. Repopulation of cancer cells during therapy: an important cause of treatment failure. Nat Rev Cancer. 2005;5:516-25.

5. Fang C, Dai CY, Mei Z, Jiang MJ, Gu DN, Huang Q. Tian L: microRNA-193a stimulates pancreatic cancer cell repopulation and metastasis through 
modulating TGF-beta2/TGF-betaRIII signalings. J Exp Clin Cancer Res. 2018; 37:25.

6. Yu Y, Tian L, Feng X, Cheng J, Gong Y, Liu X, Zhang Z, Yang X, He S, Li CY, Huang Q. elF4E-phosphorylation-mediated Sox2 upregulation promotes pancreatic tumor cell repopulation after irradiation. Cancer Lett. 2016;375: 31-8.

7. Cheng J, Tian L, Ma J, Gong Y, Zhang Z, Chen Z, Xu B, Xiong H, Li C, Huang Q. Dying tumor cells stimulate proliferation of living tumor cells via caspasedependent protein kinase Cdelta activation in pancreatic ductal adenocarcinoma. Mol Oncol. 2015;9:105-14.

8. Huang Q, Li F, Liu X, Li W, Shi W, Liu FF, O'Sullivan B, He Z, Peng Y, Tan AC, et al. Caspase 3-mediated stimulation of tumor cell repopulation during cancer radiotherapy. Nat Med. 2011;17:860-6.

9. He S, Cheng J, Sun L, Wang Y, Wang C, Liu X, Zhang Z, Zhao M, Luo Y, Tian $L$, et al. HMGB1 released by irradiated tumor cells promotes living tumor cell proliferation via paracrine effect. Cell Death Dis. 2018;9:648.

10. Feng X, Yu Y, He S, Cheng J, Gong Y, Zhang Z, Yang X, Xu B, Liu X, Li CY, et al. Dying glioma cells establish a proangiogenic microenvironment through a caspase 3 dependent mechanism. Cancer Lett. 2017;385:12-20.

11. Moschoi R, Imbert V, Nebout M, Chiche J, Mary D, Prebet T, Saland E, Castellano R, Pouyet L, Collette $\mathrm{Y}$, et al. Protective mitochondrial transfer from bone marrow stromal cells to acute myeloid leukemic cells during chemotherapy. Blood. 2016;128:253-64.

12. Samuel P, Mulcahy LA, Furlong F, McCarthy HO, Brooks SA, Fabbri M, Pink RC, Carter DRF. Cisplatin induces the release of extracellular vesicles from ovarian cancer cells that can induce invasiveness and drug resistance in bystander cells. Philos Trans R Soc Lond Ser B Biol Sci. 2018;373.

13. Becker A, Thakur BK, Weiss JM, Kim HS, Peinado H, Lyden D. Extracellular vesicles in Cancer: cell-to-cell mediators of metastasis. Cancer Cell. 2016;30:836-48.

14. Cha DJ, Franklin JL, Dou Y, Liu Q, Higginbotham JN, Demory Beckler M, Weaver AM, Vickers K, Prasad N, Levy S, et al. KRAS-dependent sorting of miRNA to exosomes. Elife. 2015:4:e07197.

15. Yan W, Wu X, Zhou W, Fong MY, Cao M, Liu J, Liu X, Chen CH, Fadare O, Pizzo DP, et al. Cancer-cell-secreted exosomal miR-105 promotes tumour growth through the MYC-dependent metabolic reprogramming of stromal cells. Nat Cell Biol. 2018:20:597-609.

16. Zeng Z, Li Y, Pan Y, Lan X, Song F, Sun J, Zhou K, Liu X, Ren X, Wang F, et al. Cancer-derived exosomal miR-25-3p promotes pre-metastatic niche formation by inducing vascular permeability and angiogenesis. Nat Commun. 2018;9:5395.

17. Au Yeung CL, Co NN, Tsuruga T, Yeung TL, Kwan SY, Leung CS, Li Y, Lu ES, Kwan K, Wong KK, et al. Exosomal transfer of stroma-derived miR21 confers paclitaxel resistance in ovarian cancer cells through targeting APAF1. Nat Commun. 2016;7:11150.

18. Toivanen R, Frydenberg M, Murphy D, Pedersen J, Ryan A, Pook D, Berman DM, Australian Prostate Cancer B, Taylor RA, Risbridger GP. A preclinical xenograft model identifies castration-tolerant cancer-repopulating cells in localized prostate tumors. Sci Transl Med, 2013. 5:187ra171.

19. Ma J, Zhang Y, Tang K, Zhang H, Yin X, Li Y, Xu P, Sun Y, Ma R, Ji T, et al. Reversing drug resistance of soft tumor-repopulating cells by tumor cellderived chemotherapeutic microparticles. Cell Res. 2016;26:713-27.

20. Valle S, Martin-Hijano L, Alcala S, Alonso-Nocelo M, Sainz B Jr. The everevolving concept of the Cancer stem cell in pancreatic Cancer. Cancers (Basel). 2018;10.

21. Vakifahmetoglu $\mathrm{H}$, Olsson $\mathrm{M}$, Zhivotovsky B. Death through a tragedy: mitotic catastrophe. Cell Death Differ. 2008;15:1153-62.

22. Menck K, Sonmezer C, Worst TS, Schulz M, Dihazi GH, Streit F, Erdmann G Kling S, Boutros M, Binder C, Gross JC. Neutral sphingomyelinases control extracellular vesicles budding from the plasma membrane. J Extracell Vesicles. 2017;6:1378056.

23. Kim MP, Fleming JB, Wang H, Abbruzzese JL, Choi W, Kopetz S, McConkey DJ, Evans DB, Gallick GE. ALDH activity selectively defines an enhanced tumor-initiating cell population relative to CD133 expression in human pancreatic adenocarcinoma. PLoS One. 2011;6:e20636.

24. Pichiorri F, Suh SS, Rocci A, De Luca L, Taccioli C, Santhanam R, Zhou W, Benson DM Jr, Hofmainster C, Alder H, et al. Downregulation of p53inducible microRNAs 192, 194, and 215 impairs the p53/MDM2 autoregulatory loop in multiple myeloma development. Cancer Cell. 2010; 18:367-81.

25. Kent LN, Leone G. The broken cycle: E2F dysfunction in cancer. Nat Rev Cancer. 2019;19:326-38.
26. Kent $L N$, Bae S, Tsai SY, Tang X, Srivastava A, Koivisto C, Martin CK, Ridolfi E, Miller GC, Zorko SM, et al. Dosage-dependent copy number gains in E2f1 and E2f3 drive hepatocellular carcinoma. J Clin Invest. 2017;127:830-42.

27. Rajamani D, Bhasin MK. Identification of key regulators of pancreatic cancer progression through multidimensional systems-level analysis. Genome Med. 2016;8:38.

28. Chang HY, Ye SP, Pan SL, Kuo TT, Liu BC, Chen YL, Huang TC. Overexpression of miR-194 reverses HMGA2-driven signatures in colorectal Cancer. Theranostics. 2017;7:3889-900.

29. Kurtova AV, Xiao J, Mo Q, Pazhanisamy S, Krasnow R, Lerner SP, Chen F, Roh $\Pi$, Lay E, Ho PL, Chan KS. Blocking PGE2-induced tumour repopulation abrogates bladder cancer chemoresistance. Nature. 2015;517:209-13.

30. Jiang MJ, Dai JJ, Gu DN, Huang Q, Tian L. Aspirin in pancreatic cancer: chemopreventive effects and therapeutic potentials. Biochim Biophys Acta. 1866;2016:163-76.

31. Zuo L, Xie Y, Tang J, Xin S, Liu L, Zhang S, Yan Q, Zhu F, Lu J. Targeting Exosomal EBV-LMP1 transfer and miR-203 expression via the NF-kappaB pathway: the therapeutic role of aspirin in NPC. Mol Ther Nucleic Acids. 2019;17:175-84

32. Hong JH, Savina M, Du J, Devendran A, Kannivadi Ramakanth K, Tian X, Sim WS, Mironova W, Xu J. A sacrifice-for-survival mechanism protects root stem cell niche from chilling stress. Cell. 2017;170:102-13 e114.

33. Bilak A, Uyetake L, Su TT. Dying cells protect survivors from radiationinduced cell death in Drosophila. PLoS Genet. 2014;10:e1004220.

34. Obenauf AC, Zou Y, Ji AL, Vanharanta S, Shu W, Shi H, Kong X, Bosenberg MC, Wiesner T, Rosen N, et al. Therapy-induced tumour secretomes promote resistance and tumour progression. Nature. 2015; 520:368-72.

35. Aubertin $K$, Silva AK, Luciani N, Espinosa A, Djemat A, Charue D, Gallet $F$ Blanc-Brude O, Wilhelm C. Massive release of extracellular vesicles from cancer cells after photodynamic treatment or chemotherapy. Sci Rep. 2016; 6:35376.

36. Saha S, Aranda E, Hayakawa Y, Bhanja P, Atay S, Brodin NP, Li J, Asfaha S, Liu $L$, Tailor Y, et al. Macrophage-derived extracellular vesicle-packaged WNTs rescue intestinal stem cells and enhance survival after radiation injury. Nat Commun. 2016:7:13096.

37. Wen S, Dooner M, Cheng Y, Papa E, Del Tatto M, Pereira M, Deng Y, Goldberg L, Aliotta J, Chatterjee D, et al. Mesenchymal stromal cell-derived extracellular vesicles rescue radiation damage to murine marrow hematopoietic cells. Leukemia. 2016;30:2221-31.

38. Tang Y, Cui Y, Li Z, Jiao Z, Zhang Y, He Y, Chen G, Zhou Q, Wang W, Zhou $X$, et al. Radiation-induced miR-208a increases the proliferation and radioresistance by targeting p21 in human lung cancer cells. J Exp Clin Cancer Res. 2016;35:7.

39. Yuan D, Xu J, Wang J, Pan Y, Fu J, Bai Y, Zhang J, Shao C. Extracellular miR1246 promotes lung cancer cell proliferation and enhances radioresistance by directly targeting DR5. Oncotarget. 2016;7:32707-22.

40. Braun CJ, Zhang X, Savelyeva I, Wolff S, Moll UM, Schepeler T, Orntoft TF, Andersen CL, Dobbelstein M. p53-responsive micrornas 192 and 215 are capable of inducing cell cycle arrest. Cancer Res. 2008;68:10094-104.

41. Kurata JS, Lin RJ. MicroRNA-focused CRISPR-Cas9 library screen reveals fitness-associated miRNAs. RNA. 2018;24:966-81.

42. Lee KH, Lee JK, Choi DW, Do IG, Sohn I, Jang KT, Jung SH, Heo JS, Choi SH, Lee KT. Postoperative prognosis prediction of pancreatic cancer with seven microRNAs. Pancreas. 2015:44:764-8.

43. Zhang J, Zhao CY, Zhang SH, Yu DH, Chen Y, Liu QH, Shi M, Ni CR, Zhu MH. Upregulation of miR-194 contributes to tumor growth and progression in pancreatic ductal adenocarcinoma. Oncol Rep. 2014;31:1157-64.

44. Chen L, Han Y, Li Y, Chen B, Bai X, Belguise K, Wang X, Chen Y, Yi B, Lu K. Hepatocyte-derived exosomal MiR-194 activates PMVECs and promotes angiogenesis in hepatopulmonary syndrome. Cell Death Dis. 2019;10:853.

45. Boo LM, Lin HH, Chung V, Zhou B, Louie SG, O'Reilly MA, Yen Y, Ann DK. High mobility group A2 potentiates genotoxic stress in part through the modulation of basal and DNA damage-dependent phosphatidylinositol 3kinase-related protein kinase activation. Cancer Res. 2005;65:6622-30.

46. Li AY, Boo LM, Wang SY, Lin HH, Wang CC, Yen Y, Chen BP, Chen DJ, Ann DK. Suppression of nonhomologous end joining repair by overexpression of HMGA2. Cancer Res. 2009;69:5699-706.

47. Martinez LA, Goluszko E, Chen HZ, Leone G, Post S, Lozano G, Chen Z, Chauchereau A. E2F3 is a mediator of DNA damage-induced apoptosis. Mol Cell Biol. 2010;30:524-36. 
48. Zelenay S, van der Veen AG, Bottcher JP, Snelgrove KJ, Rogers N, Acton SE, Chakravarty P, Girotti MR, Marais R, Quezada SA, et al. Cyclooxygenasedependent tumor growth through evasion of immunity. Cell. 2015;162:1257-70.

49. Galluzzi L, Buque A, Kepp O, Zitvogel L, Kroemer G. Immunogenic cell death in cancer and infectious disease. Nat Rev Immunol. 2017;17:97-111.

50. Hou J, Greten TF, Xia Q. Immunosuppressive cell death in cancer. Nat Rev Immunol. 2017;17:401.

\section{Publisher's Note}

Springer Nature remains neutral with regard to jurisdictional claims in published maps and institutional affiliations.

Ready to submit your research? Choose BMC and benefit from:

- fast, convenient online submission

- thorough peer review by experienced researchers in your field

- rapid publication on acceptance

- support for research data, including large and complex data types

- gold Open Access which fosters wider collaboration and increased citations

- maximum visibility for your research: over $100 \mathrm{M}$ website views per year

At $\mathrm{BMC}$, research is always in progress.

Learn more biomedcentral.com/submissions 